\title{
Gelatin Coated Albumin Nano Particles Bearing Acyclovir for Effective Management of Visceral Leishminiasis by Targeting Liver Kupffer Cells
}

\author{
Bhatt Akanksha $^{1 *}$ Bhatt Ganesh $^{1}$ Kothiyal Preeti $^{1}$ \\ ${ }^{1 *}$ Shri Guru Ram Rai Institute of Technology and Sciences, Patel Nagar, Dehradun \\ ${ }^{1}$ Shri Guru Ram Rai Institute of Technology and Sciences, Patel Nagar, Dehradun
}

\begin{abstract}
To make drug comfortable for administration to patients it is converted to dosage form by mixing with recipients, additives. Nano particles are one of the novel colloidal drug delivery systems that hold great promise for reaching the goal of controlled drug delivery as well as site-specific delivery. In the present study, an attempt was made to develop gelatin coated albumin nano particles of acyclovir for the treatment of visceral leishmaniasis with a view to see the targeting of nano particles at the required site for action and helps to provide sustain release and thus reduces the dose frequency and increase the patient compliance. Pre formulation testing is the first step in the rationale development of dosage form o a drug. The method was able to produce the free flowing nano particles. Bovine serum albumin is a biodegradable polymer for preparing targeted nano particle. FTIR studies were carried out, revealed that there was no interaction between the selected drug and polymer. The particle size analysis revealed that the particle size was found to be in the size range of $200-800 \mathrm{~nm}$, that comes under nano range. The zeta potential of nano particle was found to be -59.3 , having good stability. The maximum entrapment efficiency was found to be $86.78 \pm 0.5 \%$ for the formulation F3. The entrapment efficiency increases with increasing polymer concentration but upto a certain ratio. The maximum percentage yield was found to be $51.13 \pm 0.53 \%$ for formulation $\mathrm{F} 4$, where the concentration of albumin is highest while nano particles yield is lowest in F1 19.10 \pm 0.79 .as the concentration of albumin is lowest. In vitro release shown as, cumulative release of drug was determined up to $8 \mathrm{hrs}$ for formulationF1, F2,F3,F4 was $62.48 \pm 0.38,51.67 \pm 0.55,48.34 \pm 0.29,34.98 \pm$ 0.32 respectively. The receptor ligand binding is $95.89 \%$. And it was confirmed by checking the amount of drug release from formulation is $3.89 \%$ while prior it was $48.34 \%$, so the remaining drug was bind with receptor cells.
\end{abstract}

Keywords: Desovation Method, Receptor Ligand Binding, Zeta Potential, Pre formulation Studies.

\section{INTRODUCTION}

Drug delivery is the method or process of administering a pharmaceutical compound to achieve a therapeutic effect in humans or animals. Development of new drug molecule is expensive and time consuming. Improving safety efficacy ratio of "old" drugs has been attempted using different methods such as individualizing drug therapy, dose titration, and therapeutic drug monitoring. Current efforts in the area of drug delivery include the development of targeted delivery in which the drug is only active in the target area of the body.

Targeted drug delivery system (T.D.D.S.) is the goal oriented drug delivery in which the delivery is designed in such a way that it only signifies targeted compartment, either with the help of carrier, ligand, polymer, proteins (components through which the drug can be targeted) or by altering the molecular weight, size or shape of formulation the science of targeted drug delivery is burgeoning with new information and explosive growth of technology and methodology in this area. The delivery of drug can be done with the help of molecule that bind either with receptor or antigens targeting ${ }^{[1],[2]}$. The concept of targeting was first specified by Paul enrlich, who

\footnotetext{
${ }^{1}$ Corresponding Author: bhattakanksha261@gmail.com
} 
proposed drug delivery as magic bullets. Gregoriadls, 1981 described drug targeting using novel drug as old drug in new clothes. ${ }^{[3]}$ Colloidal drug carrier system such as micellar solutions, vesicle and liquid crystal dispersions, as well as nanoparticle dispersions consisting of small particles of $10-400 \mathrm{~nm}$ diameter show great promise as drug delivery systems ${ }^{[4]}$

\subsection{Methods of Drug Targeting}

\subsubsection{Passive Targeting}

Results in the formation of complex i.e. drug carrier complex, which avoid elimination due to body defense mechanism like metabolism, opsonization and excretion this complex will circulate in the blood and get attached to its targeted site due to property of drug. The properties are molecular weight, molecular size, nature of surface and surface charge ${ }^{[5,6]}$

\subsubsection{Inverse Targeting}

In this the RE System take colloidal carrier to achieve its targeting, where pre administration of blank colloidal carrier is done, for saturation of RES.

\subsubsection{Active Targeting}

Active targeting includes the delivery of drug through antibody, antigens, receptors and proteins. Which are either uniquely expressed or over expressed on target cell population ligand such as peptide for nevosculature targeting ${ }^{[7]}$ and

folate receptors in certain cancers. ${ }^{[8,9]}$. Antibodies have higher specificity however the production of antibodies remain expensive and time consuming and problems with stability and storage of resulting product is still exist. ${ }^{[10]}$

Nanoparticles (NPs) are one of the novel colloidal drug delivery systems that hold great promise for reaching the goal of controlled drug delivery as well as site-specific delivery. Nanoparticles are a type of colloidal drug delivery systems where the particle size ranges from 10-1000 nm in diameter. Nanoparticles are made of non-biodegradable and biodegradable polymers. The drug is dissolved, entrapped, encapsulated or attached to a nanoparticle matrix. Depending upon the method of preparation, nano particles, nano spheres or nano capsules can be obtained Nanoparticles are solid and spherical structures ranging in size and prepared from natural or synthetic polymers. A wide variety of drugs can be delivered using nanoparticles such as hydrophilic small drugs, hydrophobic small drugs, vaccines and biological macromolecules. Nano particles were able to protect drugs against chemical and enzymatic degradation and were able to reduce side effects of some active drugs ${ }^{[11-13]}$

\subsection{Preparation of Nano Particles}

The choice of preparation method essentially depends on the raw materials intended to be used and on the solubility characteristics of active compound to be associated with the particles. Regarding raw material, criteria such as biocompatibility, the degradation behavior, choice of administrative route, desired release profile of the drug and finally the type of biomedical application determine its selection.

Nanoparticles have been prepared most frequency by three methods:

Dispersion of preformed polymers

Polymerization of monomers

Ionic gelation or coacervation of hydrophilic polymers.

The internal structure of the liver is made of around 100,000 small hexagonal functional units known as lobules. Lobules are the functional unit, which made many lobes of liver. Liver consisting of various cells i.e. Hepatocytes and kupffer cells, blood vessels connected by various capillary like tubes and have larger space lined by endothelium called sinusoids. [14] 
Table1. Nanoparticle Classification

\begin{tabular}{|l|l|}
\hline Category & Examples \\
\hline Nanotubes & carbon, (fullerenes) \\
Nanowires & metals, semiconductors, oxides, sulfides \\
Nanocrystals & nitrides \\
Other nanoparticles & quantum dots insulators, semiconductors \\
& metals, magnetic materials, ceramic oxides, metals \\
Nanobots & biochip, nubots \\
\hline
\end{tabular}

\subsection{Kupffer Cell}

kupffer cells are first protective cellular line in liver sinusoid and localized in the vicinity of hepatocytes.it is a location in liver where macrophages resides, about $80-90 \%$ of macrophages are present in liver. Function of kupffer cells is ,essential in liver injury as macrophages engulf the foreign material (bacteria, virus or any infectious material),in inflammation, KCs are important source of various inflammatory-related mediators, such as poor anti-inflammatory cytokines (TNF-_, IL-6, IL-10), chemokines (MCP-2, IL-8, IP-10) and other inflammatory-related mediators (prostanoids, nitric oxide, reactive oxygen species) ${ }^{[15]}$

As discussed above those liver macrophages resides in kupffer cells and an extent to RES, so by targeting to these cells macrophages targeted automatically. Mainly targeted anti inflammatory and immunosuppressive drugs. Importance of liver macrophages, as liver has a simple structure composed of hepatic parenchymal cell and sinusoidal cell: kupffer cells (macrophages) are present in hepatic sinusoidal lumen and monocytes circulate in peripheral blood. Called blood monocytes, which are around $20 \%$ of the peripheral blood mononuclear cell (PBMC) fraction. The macrophages are professional phagocytes as having the self defense mechanism. ${ }^{[16-20]}$

Opsonization is a process by which particular antigens are rendered more susceptible to phagocytosis, foreign particle attaches to protein and prone to increase phagocytosis called opsonins, which are easily recognized by macrophages.eg of opsonins are immunoglobin ,bovine serum albumin gelatin, human fibrinocetin,bovine tuftsin, enhance phagocytosis ${ }^{21}$ ] Visceral Leishmaniasis is closely interrelated to socioeconomic, climatic, and environmental factors, the latter including land use/land cover, and topography, rainfall, temperature, and vegetation coverage ${ }^{[22]}$. As visceral leishmaniasis is a protozoa disease which infected the macrophages of body and kupffer cells (KCs) in the liver resident macrophages, which constitute $80 \%$ of tissue macrophages of the body kupffer cells. $\mathrm{KCs}$ are the first cellular protective line in liver sinusoid, which come into contact with gut derived pathogenic microbes, microbial debris and senescent erythrocytes and impede the exogenous materials by producing cytokines and directly phagocytosing and degrading them ${ }^{[23]}$

\section{MATerial AND Methodology}

\subsection{Materials}

Acyclovir (Mediflora pharmacy, Gurgoan), Bovine Serum albumin (Central drug house Ltd. New Delhi), glutarldehyde (25\%), methanol, trypsin, sodium chloride (Central drug house Ltd. New Delhi).

\subsubsection{Preparation of BSA Nanoparticles by Desolvation Method}

BSA nanoparticles were prepared by a desolvation technique. The different amount of bovine serum albumin (i.e. $50.150,250,350)$ was dissolved in sufficient amount of $0.9 \%$ Nacl, titrated to $\mathrm{pH} 7-10$. The drug was added which transformed into nanoparticles by the continuous addition of $8.0 \mathrm{ml}$ of the desolvating agent ethanol under the stirring $(500 \mathrm{rpm})$ at room temperature. After the desolvation process, few drops of $2 \%$ glutaraldehyde were added to induce particle cross linking. The cross linking process was performed under stirring of the suspension over a time period of $24 \mathrm{~h}$. 


\subsubsection{Coating of Nanopartcles}

$20 \mathrm{mg}$ of gelatin was added to $10 \mathrm{mg}$ of bovine serum albumin loaded nanoparticles which is dispersed in $5 \mathrm{ml}$ acidic phosphate buffer saline ( $\mathrm{pH}$ 5.0)and the mixture was then stirred at room temperature overnight .The resulting nanoparticles were purified by three cycles of differential centrifugation and followed by redisperesion of pellet to the original volume in Nacl. Each redespersion step was performed in an ultrasonication bath over 5 min. The solvent was evaporated and the nanoparticles were collected and stored in refrigerator at $2-8^{\circ} \mathrm{C}$

\subsection{Characterization of Nanoparticles}

\subsubsection{Shape and Size}

Morphology and size of gelatin coated nanoparticles was determined by scanning electron microscopy (SEM).

\subsubsection{Zeta Potential}

The zeta potential and surface charge of nanoparticle was determined by the zeta potential analyzer. The zeta potential of nanoparticle is commonly used to characterize the surface charge property of nanoparticles.

\subsubsection{Percentage Yield}

It is calculated to know about the efficiency of any method, thus it helps in selection of appropriate method of production.practical yield was calculated as the weight of nanoparticles recovered from each batch in relation to the sum of starting material.

\subsubsection{Entrapment Effeciency}

$200 \mathrm{mg}$ of nanoparticles were crushed in mortar and pestle $.10 \mathrm{mg}$ powder were taken and introduced in $10 \mathrm{ml}$ volumetric flask and volume makeup by phosphate buffer $\mathrm{pH}$ 7.4. $1 \mathrm{ml}$ of above solution was taken and dilute up to $10 \mathrm{ml}$.The above solution was analyzed in U.V. spectrum at $250 \mathrm{~nm}$. The entrapment efficiency of the prepared nanoparticle was calculated .

\subsubsection{In Vitro Drug Release}

In vitro drug release study was carried out by modified diffusion apparatus consists of beaker containing $50 \mathrm{ml}$ of phosphate buffer $\mathrm{pH}$ 7.4.

\subsection{Receptor Ligand Binding Study}

Goat liver was purchased from shaulter house, liver was excised and liver was washed twice with saline phosphate buffer .The organ was minced into small pieces and digested using $30 \mathrm{ml}$ of $1 \mathrm{mg} / \mathrm{ml}$ trypsin at room temperature. After putting it aside for $30 \mathrm{~min}$ the liver was homogenate and filtered to remove undigested tissue fragment. Filtrate was transferred into centrifuge tubes and centrifuge twice for $5 \mathrm{~min}$ at $300 \mathrm{rpm}$ to wash out the residual enzymatic solution. The supernatant was discarded and pellet was resuspended and differential centrifuge was performed to separate non parenchymal from parenchymal cells.After centrifuged cell suspension was kept aside for 3 min .the supernatant was put into another tube .finally the cell suspension was centrifuged at $300 \mathrm{rpm}$ for $5 \mathrm{~min}$ and supernatant was discarded and the pellet was redispersed in buffer solution. $10 \mathrm{mg}$ of nanoparticles were added into the above final solution and kept aside for $1 \mathrm{hr}$. the solution was filter after $30 \mathrm{~min}$.and filtrate was diluted and analyzed by UV spectrometer at $250 \mathrm{~nm}$.

\section{RESULTS}

\subsection{Particle size}

The SEM photomicrographs of nanoparticle are shown in Fig, shows the scanning electron microscopy of albumin nanoparticle at different magnification and area. The particle size of plane nanoparticle was found to be in the size 
American Research Journal of Pharmacy, Volume 1, Issue 1, February 2015

ISSN 2380-5706

range of 200-800 $\mathrm{nm}$. And that of gelatin coated nanoparticles were in the range of 300-900.It was observed from these photomicrographs that all samples of particles forms small cluster and are sub spherical in shape.

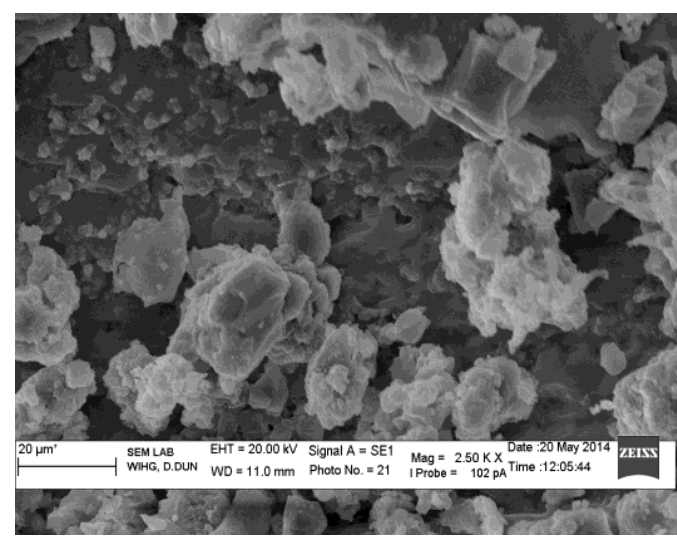

Fig1. A. Scanning electron microscopy(SEM) photomicrograph of albumin nanoparticles at $20 \mu \mathrm{m}$ area at $531 X$

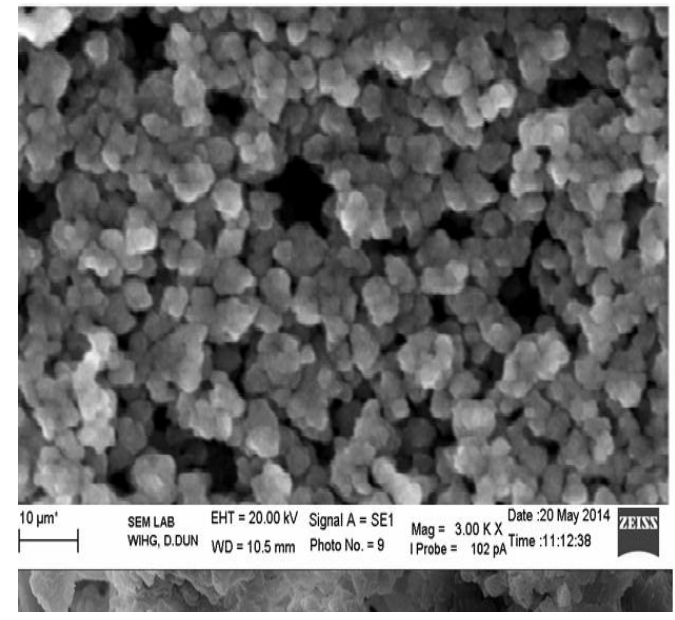

B. Scanning electron microscopy(SEM) photomicrograph of albumin nanoparticles at 10 4 m area at 3.0

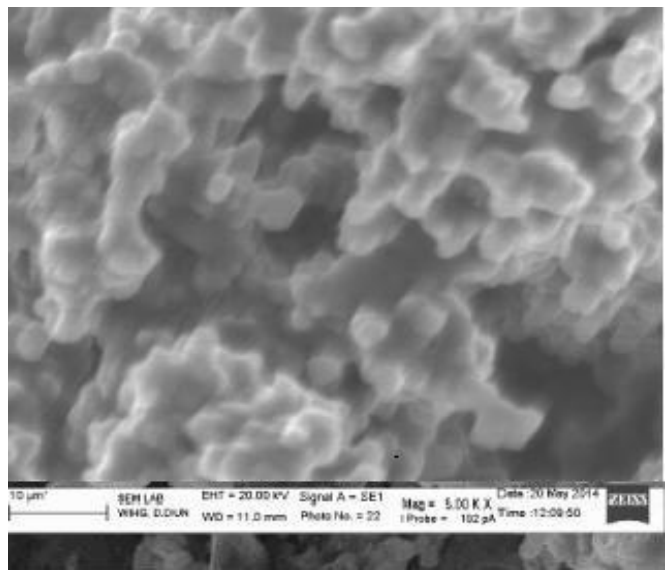

Fig2. C. Scanning electron microscopy(SEM) photomicrograph of gelatin coated albumin nanoparticles at $1 \mu \mathrm{m}$ area at $5 X$ 


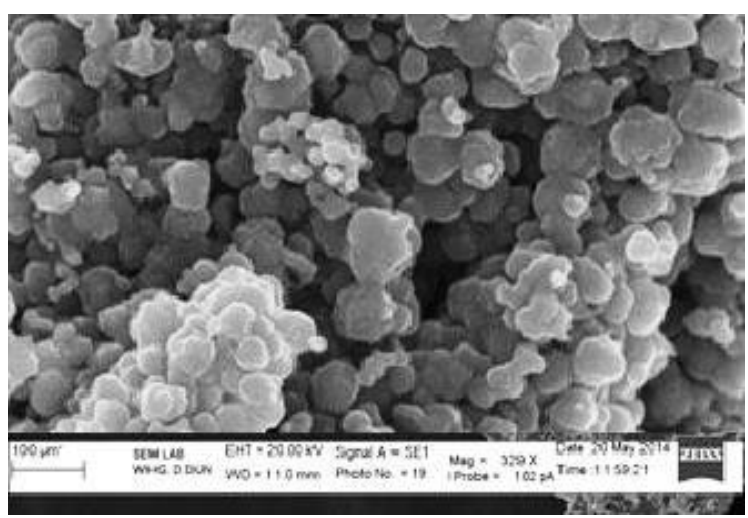

D. Scanning electron microscopy (SEM) photomicrograph of gelatin coated albumin nanoparticles at $100 \mu \mathrm{m}$ area at $329 X$

\subsection{Zeta Potential}

The graph of zeta potential of gelatin coated nanoparticles is shown in Fig 6.10. The zeta potential of nanoparticle was found to be -59.3.The gelatin coated nano particle shows the value of zeta potential -59.3 , which shows that the nanoparticle having good stability as per table 6.7 and the negative charge of the zeta potential shows that the gelatin coating will enhance the phagocytosing property of nanoparticle.

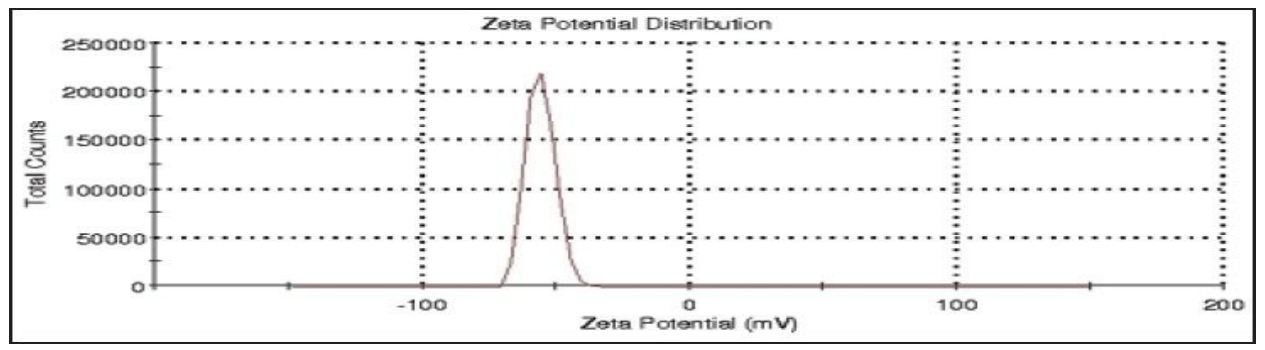

Fig3. zeta Potential of gelatin coated nanoparticle

\subsection{Percentage Yield}

The percentage yield of different formulation F1 to F4 was calculated and the yield was found to be in range of $19.10 \pm 0.79$ to $51.13 \pm 0.53 \%$.Percentage yield of all batches is shown in table 6.10.The maximum percentage yield was found to be $51.13 \pm 0.53 \%$ for formulation $\mathrm{F} 4$, where the concentration of albumin is highest while nanoparticles yield is lowest in F1 19.10 \pm 0.79 .as the concentration of albumin is lowest.

Table2. Percentage yield of gelatin coated acyclovir nanoparticles

\begin{tabular}{|l|l|l|}
\hline Formulation code & Total amount of ingridents $(\mathbf{m g})$ & Percentage yield $(\%)$ \\
\hline F1 & 570 & $19.10 \pm 0.79$ \\
\hline F2 & 670 & $21.10 \pm 0.30$ \\
\hline F3 & 770 & $34.28 \pm 0.83$ \\
\hline F4 & 870 & $51.13 \pm 0.53$ \\
\hline
\end{tabular}

\subsection{Entrapment efficiency}

The encapsulation efficiencies of all four formulations were given in table 6.9 and the entrapment efficiency was 
found to be in range of $72.34 \pm 0.45$ to $86.78 \pm 0.5 \%$. The maximum entrapment efficiency was found to be $86.78 \pm 0.5 \%$ for the formulation F3. The entrapment efficiency increases with increasing polymer concentration but upto a certain ratio.

Table3. Entrapment efficiency of gelatin coated acyclovir nanoparticle .(for $n=3)$

\begin{tabular}{|l|l|}
\hline Formulation code & Entrapment efficiency $(\boldsymbol{\%}) \pm$ S.D. \\
\hline F1 & $72.34 \pm 0.45$ \\
\hline F2 & $78.33 \pm 0.76$ \\
\hline F3 & $86.78 \pm 0.5$ \\
\hline F4 & $70.34 \pm 0.38$ \\
\hline
\end{tabular}

\subsection{In-vitro drug release}

In vitro drug release study was carried out by modified diffusion apparatus using egg membrane, consists of beaker containing $50 \mathrm{ml}$ of phosphate buffer $\mathrm{pH}$ 7.4. The dissolution study was done on all four formulations of gelatin coated nanoparticles using phosphate buffer $\mathrm{pH} 7.4$ as dissolution media. In- vitro dissolution was carried by egg membrane modified method. The readings of in-vitro permeation studies are given in Table. In vitro release is done to see how drug behaves in-vivo. The cumulative release of drug was determined up to $8 \mathrm{hrs}$ for formulation F1,F2,F3,F4 was 62.48,51.67,48.34,34.98 respectively.

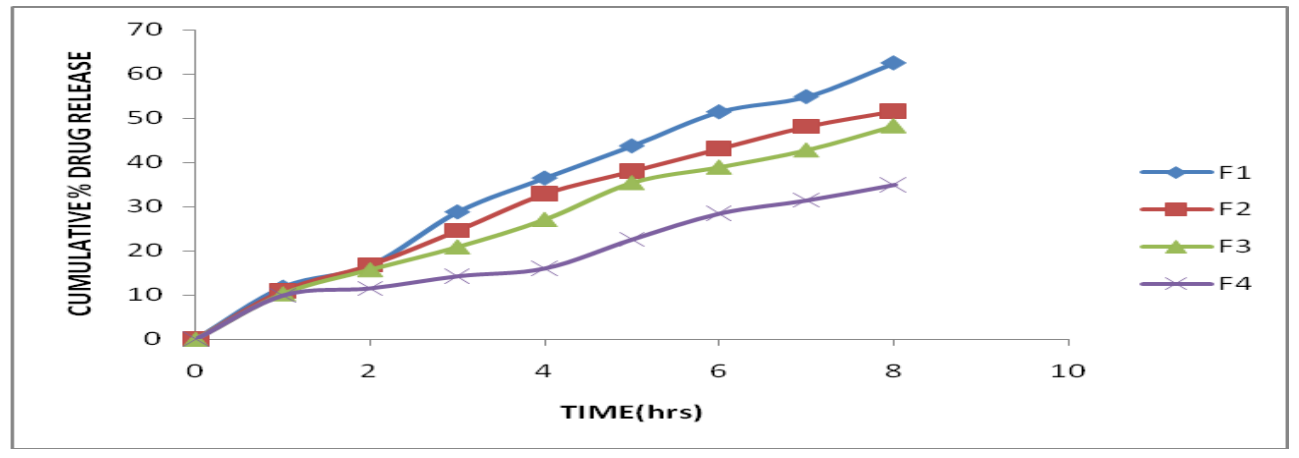

Fig3. Zero order release plot of gelatin coated nanoparticles

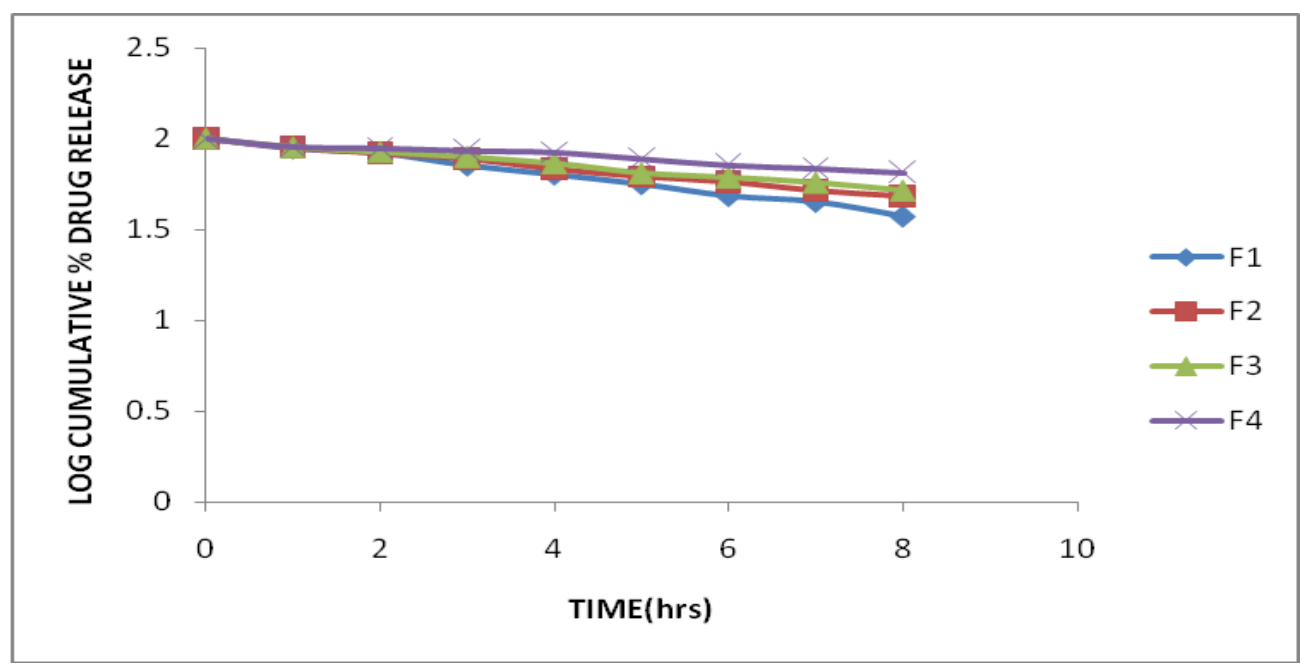

Fig4. First order release plot of gelatin coated nanoparticle 
American Research Journal of Pharmacy, Volume 1, Issue 1, February 2015

ISSN 2380-5706

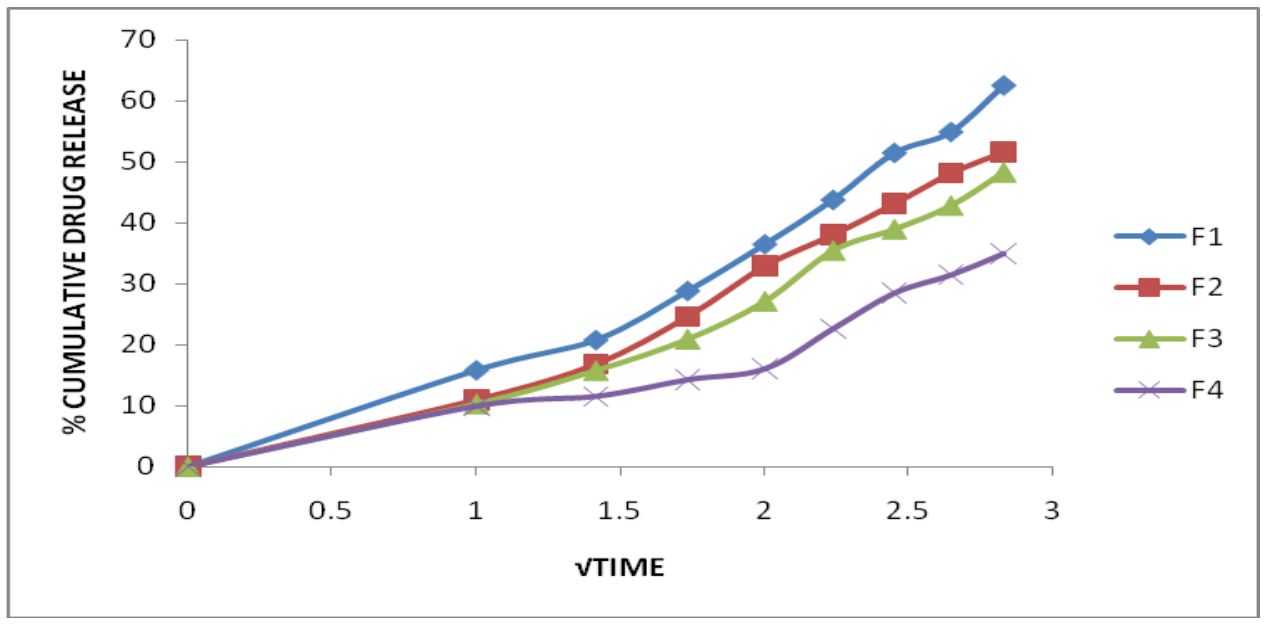

Fig5. Higuchi plot of gelatin coated nanoparticle

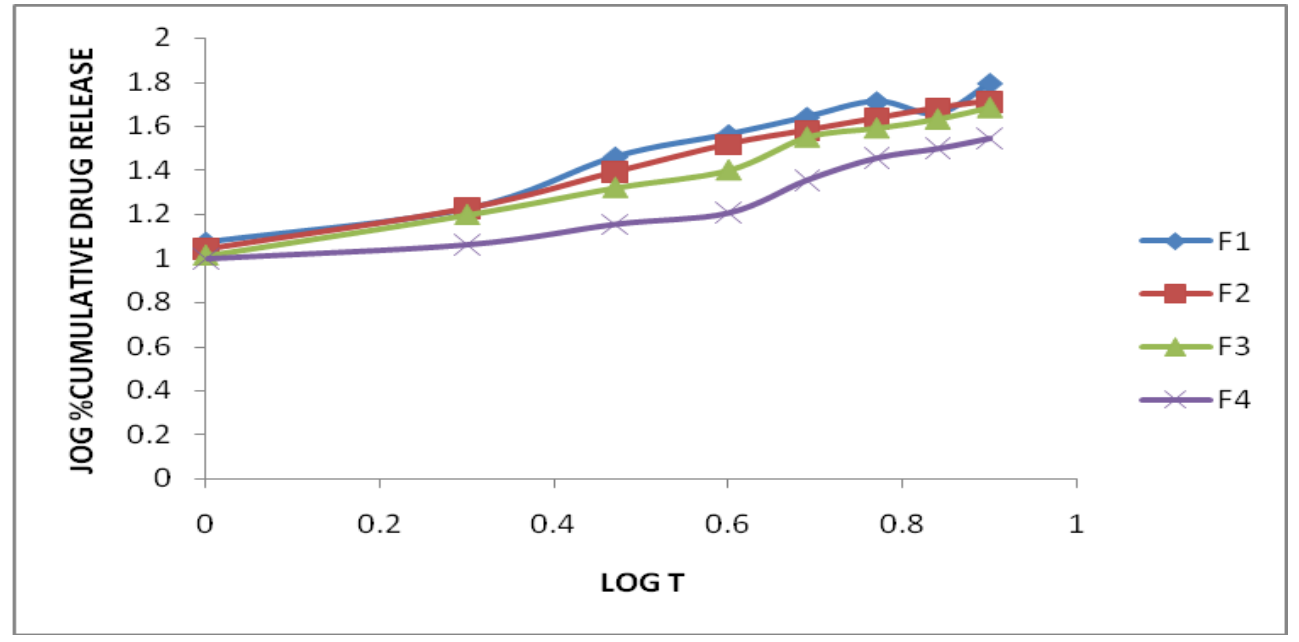

Fig6. Korsmeyer Peppa's plot of gelatin coated nanoparticle

\section{Mathematical Modeling}

The data obtained from in-vitro release study was treated by various convectional mathematical models to determine the release mechanism from designed nano particle formulations .selection of suitable release model was based on values of R.(correlation coefficient), $\mathrm{k}$ (release constant and $\mathrm{n}$ (diffusion exponent) obtained from curve fitting of release data. The drug release mechanism and kinetics are two important characteristics of delivery system for the drug dissolution profile based on mathematical models .

Mathematical Models are:

Zero order model

First order model

Higuchi`s square root of time equation model

Korsmeyer-peppas power law equation model 
The data obtained from in- vitro release study was treated by various mathematical models (zero order ,first order ,higuchi and korsmeyer peppa's) to determine the release mechanism from designed nanoparticle formulation .selection of a suitable release model was based on the values of $\mathrm{R}$ (correlation coefficient ) and $\mathrm{k}$ (release constant) and $\mathrm{n}$ (diffusion exponent) obtained from curve fitting of release data.

It was found that all formulations follow first order kinetics.

The regression coefficient for all formulation of Higuchi plot was found to be almost linear, suggesting that the formulation was diffusion controlled. Korsmeyer peppas model was used when the release mechanism is not well known or when more than one type of phenomenon is involved.The value of $n$ is used to characterize the different release mechanism. The value of $\mathrm{n}$ for $\mathrm{F} 1$ to $\mathrm{F} 4$ formulation is found in between 0.65 to 0.81 , that all the formulation indicates that the release is non-Fickian diffusion method.

Table5. Model fitting release profile of formulations F1 to F4

\begin{tabular}{|l|l|l|l|l|}
\hline Formulation code & \multicolumn{3}{|l|}{ Regression $\operatorname{coefficient}\left(\mathbf{R}^{\mathbf{2}}\right)$} & Slope (n) \\
\hline & zero order & first order & Higuchi's & korsmeyer peppa's \\
\hline F1 & 0.987 & 0.994 & 0.965 & 0.811 \\
\hline F2 & 0.981 & 0.996 & 0.964 & 0.780 \\
\hline F3 & 0.987 & 0.995 & 0.955 & 0.769 \\
\hline F4 & 0.972 & 0.973 & 0.921 & 0.651 \\
\hline
\end{tabular}

\section{RECEPTOR LIGAND BINDING STUDY}

By considering the absorbance of formulation F3 at U.V. $(250 \mathrm{~nm})$ it was found that the receptor ligand binding is $95.89 \%$. And it was confirmed by checking the amount of drug release from formulation is $3.89 \%$ while prior it was $48.34 \%$, so the remaining drug was bind with receptor cells.

\section{DISCUSSION}

In the present study, an attempt was made to develop gelatin coated albumin nanoparticles of acyclovir for the treatment of visceral leishmaniasis with a view to see the targeting of nanoparticles at the required site for action and helps to provide sustain release and thus reduces the dose frequency and increase the patient compliance. Nanoparticles were successfully prepared by desolvation method.The method was able to produce the free flowing nanoparticle. Bovine serum albumin is a biodegradable polymer for preparing targeted nanoparticle .FTIR studies were carried out to find out the possible interactions between the drug and polymer. The study revealed that there was no interaction between the selected drug and polymer. The particle size analysis revealed that the particle size was found to be in the size range of 200-800 nm.that comes under nano range. The zeta potential of nanoparticle was found to be -59.3 , which shows that the nanoparticle having good stability, the negative charge of the zeta potential shows that the gelatin coating will enhance the phagocytosing property of nanoparticle. The maximum entrapment efficiency was found to be $86.78 \pm 0.5 \%$. For the formulation F3. The entrapment efficiency increases with increasing polymer concentration but up to a certain ratio. The maximum entrapment efficiency was found to be $86.78 \pm 0.5 \%$ for the formulation F3. The entrapment efficiency increases with increasing polymer concentration but upto a certain ratio The maximum percentage yield was found to be $51.13 \pm 0.53 \%$ for formulation $\mathrm{F} 4$, where the concentration of albumin is highest while nanoparticles yield is lowest in F1 19.10 \pm 0.79 .as the concentration of albumin is lowest. In vitro release shown as, cumulative release of drug was determined up to $8 \mathrm{hrs}$ for formulation F1, F2, F3, F4 was $62.48,51.67,48.34,34.98$ respectively. From result it was concluded that on increasing the concentration of polymer release rate of formulation decreased. The receptor ligand binding is $95.89 \%$. And it was confirmed by checking the amount of drug release from formulation is $3.89 \%$ while prior it was $48.34 \%$, so the remaining drug was bind with receptor cells. 


\section{ACKNOWLEDGEMENTS}

It take this privilege and pleasure to acknowledge the contributions of many individuals who have been inspirational and supportive throughout my work undertaken and endowed me with the most precious knowledge to see success in my endeavor. , I am grateful to Dr.Ganesh Kumar Bhatt M.Pharm. Associate Professor for his invaluable guidance and untiring supervision which enable me in successful completion of my project work.

\section{REFERENCE}

[1] Oberhauser ,B; Mechtler, K.; Koch, C.; and Wagner, E.; 1994,The influence of endosome-disruptive peptides on gene transfer using synthetic virus-like gene transfer system .J Biol chem,269,12918-12924.

[2] Plank, C; Zauner, W; and Wagner, E.;1998, Application of membrane-active peptides for drug and gene delivery across cellular membranes.Rev 34(1), 21-35.

[3] Vyas, S.,P;Targeted and controlled drug delivery; novel carrier system.rev,( 1)38-79.

[4] P.,Dwarakanadha ;Reddy;D.,Swarnalatha; 2010, Recent Advances in Novel Drug Delivery Systems, Int.J. PharmTech Res,2(3),2025-2027.

[5] Frankel; Alan, D; 1988;Cellular uptake of tat protein from human immunodeficiency virus,Cell, 55,1189-1193.

[6] Maeda, H.; 2010,Tumor-selective delivery of macromolecular drugs via the EPR effect: background and future prospects, Bioconjugate Chem., 21(5),797-802.

[7] Planck, C.;Zauner W.; Wagner, E.;1998,application of membrane active peptides for drug and gene delivery across cellular membrane, Adv. Drug delivery Rev.,21-35.

[8] E.,Wagner; C., Plank; K., Zatloukal;M., Cotten; and M., L., Birnstiel, 1992,Influenza virus hemagglutinin HA-2 N-terminal fusogenic peptides augment gene transfer by transferrin-polylysine-DNA complexes: toward a synthetic virus-like gene-transfer vehicle., Proc Natl Acad Sci U S A, 89(17),7934-7938.

[9] Nadeasdi, L.,Subabrao; N.,K.,Parenete; R,.A.,Szoaka; F.,C., 1990,Association of pH sensitive peptide with membrane vesicles :role of amino acid sequence ,J Biochem ,29,48-57

[10] Gottschalk ,S;Sparrow, J.,T., Hauer, J, Mims, M.P.; Leland F.E.; Woo S.,L.; Smith, L.,C.; 1996,A novel DNA-peptide complex for efficient gene transfer and expression in mammalian cells., Gene Ther,3(5),448-57.

[11] Krishna, R.,S.,M.; Shivakumar, H.,G.; Gowda, D.,V.; and Banerjee,S. 2005,Nanoparticles : A Novel Colloidal Drug Delivery System, Indian J.Pharm. Educ. Res.,40(1),15-21

[12] V.,J.; Mohanraj, and Y, Chen;2006 ,Nanoparticles - A Review, Tropical Journal of Pharmaceutical Research, 5 (1), $561-573$

[13] Fabienne, Danhier ;Eduardo, Ansorena ; Joana M., Silva ; Régis, Coco ;Aude, Le Breton;Véronique ,Préat;2012, PLGA-based nanoparticles: An overview of biomedical applications, Journal of Controlled Release, 161,505-522

[14] Tortora .Principles of anatomy and physiology.8th edition,777-781

[15] Qiao ,Yang;Yu ,Shi; Jiliang He and Zhi, Chen;2012,The evolving story of macrophages in acute liver failure, Immunology letters. 147, 1-9.

[16] Klaas, poelstra; Jai ,Prakash; and Leonie, Beljaars; 2012, Drug targeting to diseased liver, Journal of controlled release. 161:188-197.

[17] Mark,Barnes;Laura,J.;Dixon; Zhang-Xu, Liu; Hui; Tang; Laura and E., Nagy;2013,Macrophages and Kupffer Cells in Drug-Induced Liver Injury ,Elsevier Inc..147-153 
American Research Journal of Pharmacy, Volume 1, Issue 1, February 2015

ISSN 2380-5706

[18] Kiyoshi ,Takahashi.; 2002 , Development and differentiation of macrophages and related cells: Historical view and current concepts, Journal of clinical and experimental hematopathology, 41,1-22.

[19] Qureshi, M. A.; 1998,Role of Macrophages in Avian Health and Disease ,Poultry Science,77,978-982.

[20] Cornelia; S. ,Viebahn; 2010,Invading macrophages play a major role in the liver progenitor cell response to chronic liver injury, Journal of Hepatology. 53,500-507

[21] Groves, E.;2008,Molecular mechanism of phagocytic uptake in mammalian cell, Cell molecular life science.65,1957-76.

[22] Gouri, Sankar ,Bhunia;shreekant, keshari; Nandini, Chatterjee;Vijay ,Kumar; and Pradeep, Das; 2013.The Burden of Visceral Leishmaniasis in India Challenges in Using Remote Sensing and GIS to Understand and Control. ISRN Infectious Diseases, 1-14.

[23] Ciara, Kelly; 2011. Caroline Jefferies and Sally-Ann Cryan.,Targeted Liposomal Drug Delivery to Monocytes and Macrophages, Journal of Drug Delivery.2010,1-11. 